\title{
Novel Gelatin-Siloxane Nanoparticles Multiple Modified by Cationic Peptide and Aptamers as Efficient Gene Vector
}

\author{
Xin-Hua Tian ${ }^{1 *}$, Yu-Hua Wang ${ }^{1}$, Feng $\mathrm{Wei}^{1}, \mathrm{Xiao-Ning} \operatorname{Lin}^{1}$ and Lei Ren ${ }^{2,3}$ \\ ${ }^{1}$ Department of Neurosurgery, Zhongshan Hospital, Xiamen University, Xiamen, China \\ ${ }^{2}$ Research Center of Biomedical Engineering, Department of Biomaterials, College of Materials \\ ${ }^{3}$ State Key Laboratory for Physical Chemistry of Solid Surfaces, Xiamen University, Xiamen, China \\ Email: txhmd@163.com
}

\begin{abstract}
An excellent gene vector is of great importance for the development of the gene therapy. Gelatin-siloxane nanoparticles (GS NPs) with controlled size and surface charge were synthesized through a two-step sol-gel process for gene vector. In order to increase the efficiency of tumour targeting, HIV-derived Tat peptide and TTA1, a kind of modified RNA aptamer, were further grafted onto GS NPs (GS-PEG-TTA1/TAT NPs). It was demonstrated in this article that gelatin-siloxane nanoparticles (GS NPs) have the significant characteristic of being innocuous, small, covered with positive charge and even have been modified with PEG, TTA1, and TAT. The modified nanoparticles, GS-PEG-TTA1/TAT, not only have the ability of crossing the cell membrane with DNA accompanied effectively, but also could target the U251 cells and impel the DNA express right in the cells with the help of aptamer TTA1.
\end{abstract}

Keywords: Nanoparticles, peptide, blood-brain barrier, brain targeting

\section{Introduction}

Glioblastoma with a high mortality is one of the most aggressive malignant primary brain tumors in humans and has dismal survival of less than a year, despite advances in imaging techniques and treatment with surgery and radiation[1,2]. The main reason is the difficulty in delivering drugs across the blood-brain barrier (BBB) to the brain. The unique structural characteristics of the BBB protect neurons and preserve the homeostasis of the central nervous system (CNS), but at the same time, BBB represents an insurmountable obstacle for a large number of drugs, including antibiotics and neuropeptides[3]. As the significant reason that most therapeutic agents cannot cross the BBB, few treatments are available against disorders in CNS.

Recent years, some scholars point that gene therapy may be potential for curing both genetic and acquired disease[4,5]. Gene therapy, defined as the delivery of nucleic acids into the recipient's cells for therapeutic purposes, could have significant potential in the treatment of various CNS diseases[6].Although there is some advances in the field, delivery of nucleic acids to target cells and tissues with high-efficiency is still challenging. Synthetic, non-viral vectors are potential alternatives to viral vectors, and may overcome several problems encountered in viral vector-mediated therapy, including immune responses, limited DNA carrying capacity, recombination and high cost. Non-viral vectors, however, suffer from the problem of the relatively low efficiency of gene delivery, particularly for in vivo applications [7].

Therefore, there is great importance to excogitate a novel gene delivery system, which not only has low toxicity, small particle size, low immunogenicity, but also can effectively enhance the delivery of therapeutic gene for penetrating the BBB and targeting the lesions exactly.

Among the various strategies proposed to circumvent the blood-brain barrier in recent years, nanobiotechnology is taking a more and more important place. It was demonstrated that nanoparticle systems could effectively carry various drugs crossing the BBB[8,9]. Furthermore, because of their low toxicity, small particle sizes, and appropriate surface modification, nanoparticles can easily cross the blood 
capillaries and arrive at the diseased region.

The cell-penetrating peptides (CPPs) are typically short cationic sequences and may be derived from natural sources or be synthetically designed constructs[10]. The CPPs have great ability of delivering variety of macromolecules, such as therapeutic drugs, oligonucleotides, proteins and so on. Though the mechanism of them not very clearly, they do have the capacity of helping the drugs across the plasma membrane. There were already some study proved that when modified by the Cationic CCPs and poly (ethylene glycol) (PEG), gelatin-siloxane nanoparticles (GS NPs) had more excellent capacity of penetrating the BBB and residing longer in the Blood Circulation System [8].

Aptamers are small oligonucleotides, which can be used to develop compounds with favorable pharmacokinetic properties, such as being small in size, lacking immunogenicity, ease of synthesis and high binding affinity $[11,12]$. As a kind of high utilization molecular probe, they have high affinity and selectivity to their cancer-specific biomarkers, such as receptor tyrosine kinase (RTK), vascular endothelial growth factor (VEGF), tenascin-C (Tnc), nucleolin, mucin-1 and prostate-specific membrane antigen (PSMA)[13]. TTA1 (5'-CCTGCACTTGGCTTG-

GATTTCAGAAGGGAGACCC-3'), a kind of modified RNA aptamer, has an unique conjugation site which has special affinity for tenascin-C. Tenascin-C is a large extracellular glycoprotein. It is unique in its distinct pattern of expression. Little or no tenascin-C is detected in healthy adult tissues[14]. Its exuberant expression appears to play an important role in tumor progression by regulating cell adhesion and migration, such as carcinomas of the lung, breast, prostate, and colon, as well sarcomas, glioblastomas, and melanomas [15].

The aim of this experiment is to construct a kind of nanoparticle gene carrier system, which was modified by cationic peptides TAT and aptamers TTA1. The experiment is devised to confirm whether the novel genonic vector can cross the cell membrane successfully and target the tumor cell more efficiently. Whether it can provide basis for gene therapy with penetrating the blood-brain barrier and targeting treatment of spongiocytoma should be considered serious-minded as well.

\section{Materials and Methods}

\subsection{Materials}

Gelatin (bloom number 240-270, pH 4.5-5.5) was purchased from BBI (Madison, WI). 3Glycidoxypropyl-trimethoxysilane(GPSM) and 3-aminopropyl-trimethoxysilane (APTMS) were purchased from Acros Organics (Fair Lawn, NJ). N-succinimidyl-3-(2-pyridyldithio) propionate (SPDP) was purchased from Pierce Biotechnology Inc (Rockford, IL). 1-[3-(dimethylamino)propyl]-3ethylcarbodiimide (EDC) and N-Hydroxysuccinimide (NHS) were provided by Chinese GL Biochem Ltd, (Shanghai, China). NH2-PEG ${ }_{2000}-\mathrm{COOH}$ was provided by Chinese Beijing Kai Zheng Biotech Development Co, (Beijing, China). TAT peptide (YGRKKRRQRRR) and fluorescein isothiocyanate (FITC)-labeled TAT peptide were provided by the Chinese Peptide Co, (Hangzhou, China). Aptamer TTA1 (5'-CCTGCACTTGGCTTGGATTTCAGAAGGGAGACCC-3') and fluorescein isothiocyanate(FITC)labeled TTA1 were purchased from Sangon biotech (Shanghai) CO,(Shanghai China). Dulbecco's Modified Eagle's Medium (DMEM), fetal bovine serum (FBS), and penicillin-streptomycin were obtained from Hyclone (Utah, USA). Luciferase plasmid pGL3 and luciferase assay system were purchased from Promega (Madison, USA). Dimethyl sulfoxide(DMSO) were purchased from Sigma-Aldrich (Beijng, China). Endotoxin-free plasmid purification kit was purified from Qiagen (Dus, Germany). All materials used were of analytical grade and used without further purification.

\subsection{Preparation of Plasmid}

Luciferase plasmid pGL3 was transformed in Escherichia coli DH5- $\alpha$ and amplified in Lysogeny broth medium at $37^{\circ} \mathrm{C}$ overnight. After that it was purified using the Qiagen endotoxin-free plasmid purification kits according to the manufacturer's instruction. The concentration of the plasmid was quantified by the measurement of UV absorbance at $260 \mathrm{~nm}$ using a Gene Ray UV Photometer (Biometra). The plasmid was preserved at $-20{ }^{\circ} \mathrm{C}$ when unused. 


\subsection{Preparation of Nanocomplexes Gene Vector}

The general process of preparing and detecting of multiple modified nanocomplexes gene vector was described in reference [16,17]. Briefly, $0.15 \mathrm{~g}$ gelatin was solubled in $20 \mathrm{ml}$ hydrochloric acid (pH 3.0) and stirred for $20 \mathrm{~min}$ in $60^{\circ} \mathrm{C}$. Then $200 \mu \mathrm{g}$ GPSM was add into the gelatin solution and stirred for $30 \mathrm{~min}$. Generation of gelatin-siloxane nanoparticles was associated with the introduction of APTMS into the above mixture. After purified by centrifugation $\left(14,500 \mathrm{rpm}, 30^{\circ} \mathrm{C}\right.$, and $\left.12 \mathrm{~min}\right)$ and re-dispersed in $\mathrm{pH} 8.0$ phosphate-buffered solution. EDC and NHS were used in the progress of NH2- $\mathrm{PEG}_{2000}-\mathrm{COOH}$ being linked to the surfaces of GS NPs as coupling reagents. After sulfhydrylated via SPDP, the PEGylated GS NPs was ready to be modified by peptides and aptamer. TTA1 with a free sulfhydryl group at the end of the strand was coupled to the PEGylated nanoparticles via a disulfide bond after stirred in the solution for 4 h. Finally, the nanocomplexes were synthesized by mixing the GS NPs with the peptides TAT having free sulfhydryl group at the end of the strand. Through a series of process, the GS-PEG NPs, GS-PEG-TTA1 NPs, and GS-PEG-TTA1/TAT NPs were obtained. In order to get the compounds of DNA loaded with nanoparticles, nanoparticles stirred with luciferase plasmid DNA pGL3 at the ratio of 100:1 (w/w) for 1 min and then static cultivation at room temperature for $1 \mathrm{~h}$.

\subsection{Cell Culture}

Human glioblastoma U251cell, purchased from Chinese Academy of Sciences type culture collection cell library committee (shanghai, China), was maintained in DMEM, supplemented with $10 \%$ FBS and $1 \%$ penicillin/streptomycin in a humidified atmosphere $\left(5 \% \mathrm{CO}_{2}, 37^{\circ} \mathrm{C}\right)$. The old mediums were replaced every 2 days. When covered the cell cultures dishes over $80 \%$, the cells were digested with trypsin solution $(0.125 \%, \mathrm{w} / \mathrm{v})$ and separated.

\subsection{Characterization of Nanoparticles}

In the synthetic process of the gene vector, FITC labeled TAT and TTA1 were used. The products, GS-PEG-TTA1-FITC NPS and GS-PEG-TTA1/TAT-FITC NPS, were used to confirm whether TTA1 and TAT had been linked to the GS NPs. The nanoparticles and their third time liquid supernatant obtained during the process of purifying the nanocomplexes by centrifugation were used to test fluorescence spectrum via a Fluorescence spectrophotometer (Hitachi, Japan) at room temperature. Origin8 analysis software was used for data processing. The measured data was integrated to compare the distinction between the materials and the supernatants.

The synthesized nanoparticles were suspended by deionized water at a concentration of $0.1 \mathrm{mg} / \mathrm{ml}$. The particle size and surface charge of the gene vectors, constituted by GS-PEG NPs, GS-PEG-TTA1 NPs and GS-PEG-TTA1/TAT NPs, were determined by a Nano-ZS zetasizer dynamic light scattering detector (Malvern Instruments, UK), respectively. For reproducibility, each group was measured at least 5 samples and each sample was measured at least 3 times.

The nanoparticles, GS-PEG-TTA1 NPs and GS-PEG-TTA1/TAT NPs, were treated in the same way as above and were dropped onto a copper grid. After dried in the air, they were carried out for analysis by transmission electron microscopy (2100 HC, Japan) an operating voltage of $200 \mathrm{kV}$ in bright-field mode.

\subsection{Cytotoxicity Assay in Vitro}

The cytotoxicity of the NPs was evaluated by MTT assay. U251 cells were digested and planted into the 96-well plates at a density of $5 \times 10^{3}$ cells/well. After incubated at $37^{\circ} \mathrm{C}$ with $5 \% \mathrm{CO}_{2}$ for $24 \mathrm{~h}$, the cells were co-cultured with GS-PEG NPs, GS-PEG-TTA1 NPs and GS-PEG-TTA1/TAT NPs with the nanocomplexes diluted by $100 \mu \mathrm{L}$ serum-free medium at the different concentrations $(100,300,500 \mu \mathrm{g} / \mathrm{mL})$ and incubated with U251 cells for $24 \mathrm{~h}$. Each concentration was set 6 same samples as reiteration. The control group, which was not exposed to nanoparticles, was incubated in the same environment as above. Then old medium was removed and the cells were incubated in fresh medium for a further 4 hours at $37{ }^{\circ} \mathrm{C}$ in a $5 \% \mathrm{CO}_{2}$ atmosphere after $20 \mu \mathrm{L}$ MTT ( $5 \mathrm{mg} / \mathrm{mL}$ in phosphate-buffered saline) was added to each well. After removed the MTT and the medium, $100 \mu \mathrm{L}$ of dimethylsulfoxide was added into the plates to dissolve the crystals formed by living cells. The absorbance at $570 \mathrm{~nm}$, used to evaluate the cell viability, was 
determined by a spectrophotometric microplate reader (Biotek ELX800, Winooski). The relative cell viability (\%) was expressed as a percentage in comparison with control cells.

\subsection{In Vitro Cellular Uptake of Nanoparticles}

In order to confirm whether the nanoparticles modified by TTA1 and TAT can penetrate the cell membrane efficiently and have an excellent advantage of targeting the tumor cell, the uptake experiment was designed.

\subsubsection{Analysis by Fluorescence Microscope}

The U251cells were seeded in the glass bottom dishes at a density of $2 \times 10^{5}$ cells/well and co cultured with NPs/DNA (100 $\mu \mathrm{g}$ nanoparticles/dish). In this experiment, nanoparticles were labeled by FITC and DNA was labeled by Propidium iodide (PI) in serum-free DMEM medium. After incubated at $37^{\circ} \mathrm{C}$ with $5 \% \mathrm{CO}_{2}$ for $4 \mathrm{~h}$, the cells were fixed for $30 \mathrm{~min}$ with $4 \%$ paraformaldehyde in room temperature. Then rinsed with PBS for 3 times and stained with DAPI $(5 \mu \mathrm{g} / \mathrm{mL})$ for 20 min at $37{ }^{\circ} \mathrm{C}$. The stained cells were

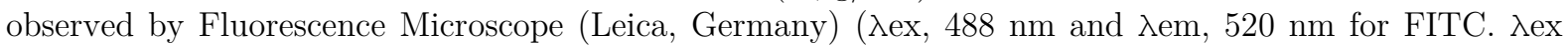
$535 \mathrm{~nm}$ and $\lambda \mathrm{em}, 615 \mathrm{~nm}$ for PI) and the images were analyzed with Olympus CLSM software.

\subsubsection{Quantification analysis of GS NPs by flow cytometry}

U251 cells $\left(2.0 \times 10^{5}\right)$ were seeded in 24 -well plates and cultured for $24 \mathrm{~h}$. After rinsed carefully, the cells were incubated with $100 \mu \mathrm{g} / \mathrm{ml}$ GS/DNA suspension in serum-free medium for $1 \mathrm{~h}$ and $2 \mathrm{~h}$. The cells were washed with PBS twice, digested by trypsin and collected in centrifugal tube. For determining the internalization effect of the nanoparticles, EPICS XL flow cytometer (Beckman Coulter, USA) was used, by which the red emitting PI incorporated in GS/DNA serves as a marker to quantitatively $(\lambda \operatorname{ex~} 535 \mathrm{~nm}$ and $\lambda \mathrm{em}, 615 \mathrm{~nm}$ for PI).

\subsection{In Vitro Transfection Analysis}

U251cells and Hela cells were seeded in 24 well plates at the same density of $4 \times 10^{5}$ cells/well and maintained in DMEM medium, respectively. NPs/DNA were added into the plates at the concentrations of $100 \mathrm{\mu g} / \mathrm{ml}$ with $1 \mathrm{~m} / \mathrm{L}$ serum-free medium and incubated for $8 \mathrm{~h}$. The old medium was removed and another $1 \mathrm{ml}$ medium containing 10\% FBS was added. The cells were incubated with complexes for other 24 hours at $37{ }^{\circ} \mathrm{C}$ under a $5 \% \mathrm{CO}_{2}$ atmosphere, followed by washing with sterile PBS and then harvested with $0.1 \%$ Triton X100 and centrifuged $\left(12,500 \mathrm{rpm}, 4{ }^{\circ} \mathrm{C}, 2\right.$ minutes $)$. The supernatant was reserved to be used to measure the light emission by a FB12 luminometer (Berthold, Germany). BCA protein assay kit was applied for determining the protein concentration. The ratio of relative light units (RLU)/mg protein was considered as the percentage composition of luciferase.

\subsection{Statistical Analysis}

Results were represented as means \pm standard deviation. All measurements were collected in triplicate. Statistical testing was carried out using the one-way ANOVA test. A difference was considered statistically significant if the $p$ value is $<0.05$.

\section{$3 \quad$ Results}

\subsection{Structure Confirmation and Biochemical Characterizations of Nanoparticles}

The fluorescence spectrum was employed to confirm whether TAT or TTA1 was linked to GS NPS successfully. The comparison between the samples and supernatants is shown in Figure 1. It indicates that both GS-PEG-TTA1/TAT NPs and GS-PEG-TTA1 NPs have higher fluorescence intensity (4500 a.u, 110000 a.u than their supernatants(500 a.u,18000 a.u), respectively. The results can explicitly prove that the protein and aptamer were linked to the NPs exactly. And as shown in Fig.2, transmission electron microscopy (TEM) demonstrated that the GS-PEG-TTA1/TAT NPs and GS-PEG-TTA1 NPs both had the characteristics of average size, dispersity and sphericity. Just as described by references [6]. In addition, 
the sizes and surface charge were examined with Nano-ZS zetasizer dynamic light scattering detector (Fig.3). The results showed that the surface charge values of GS, GS-PEG, GS-PEG-TTA1, and GS-PEG-TTA1/TAT NPs were $(25.12 \pm 1.78) \mathrm{mV},(3.12 \pm 0.46) \mathrm{mV},(3.33 \pm 0.21) \mathrm{mV},(30.32 \pm 3.37) \mathrm{mV}$ respectively. The average diameters of GS, GS-PEG, GS-PEG-TTA1, and GS-PEG-TTA1/TAT NPs were $(170.95 \pm 2.90) \mathrm{nm},(192.15 \pm 2.05) \mathrm{nm},(231.6 \pm 0.28) \mathrm{nm},(250.45 \pm 4.60) \mathrm{nm}$, respectively. It is indicated from Fig.3 that when adding plasmid into nanoparticles suspension, the diameter increased and the surface charge decreased, especially in the GS NPs group and GS-PEG-TTA1/TAT NPs group.
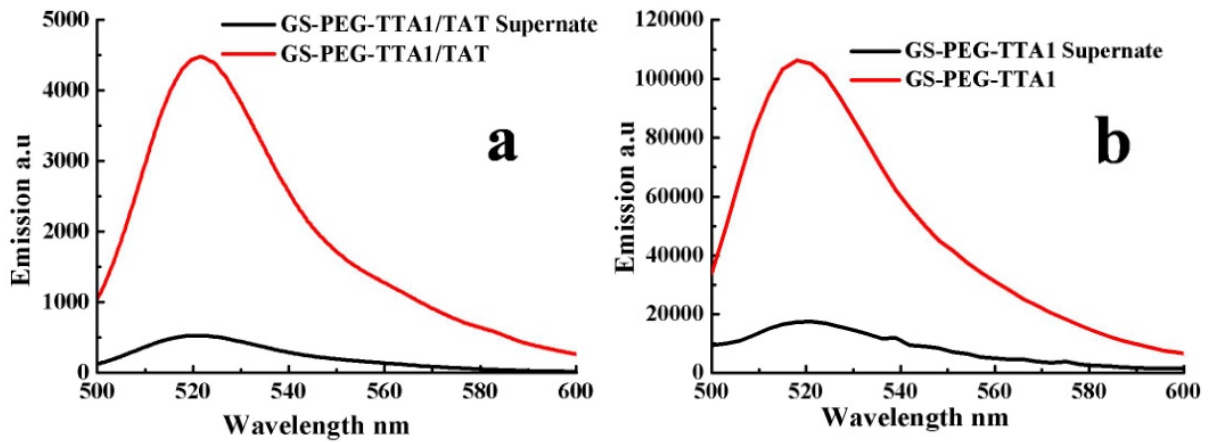

Figure 1. The fluorescence spectrum of (a)GS-PEG-TTA1/TAT-FITC NPs and (b)GS-PEG-TTA1-FITC NPs.
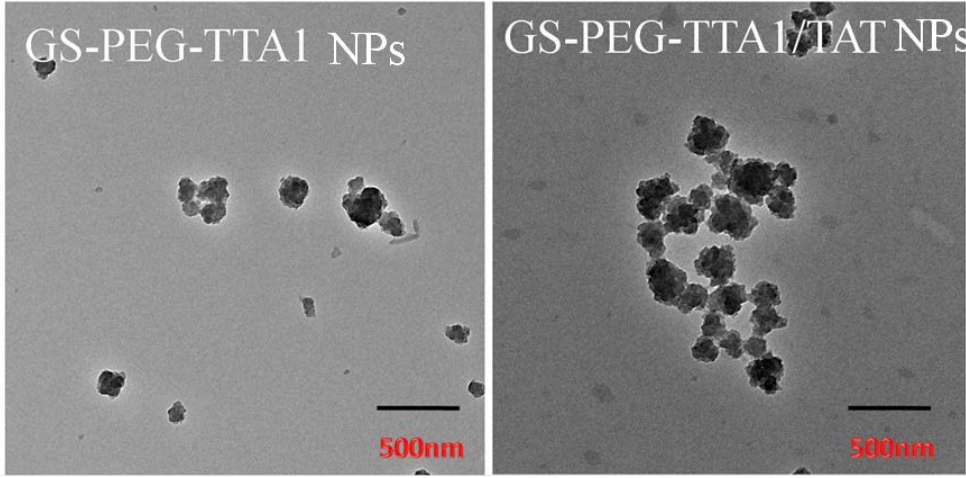

Figure 2. The TEM image of GS-PEG-TTA1 NPs and GS-PEG-TTA1/TAT NPs.

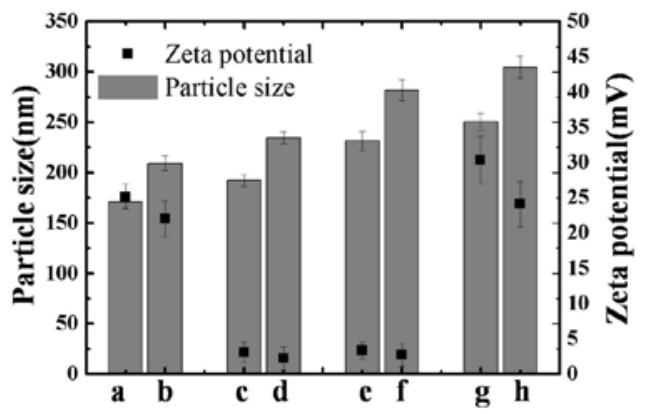

Figure 3. The size and surface charge of (a)GS, (b) DNA/GS, (c) GS-PEG, (d) DNA/GS-PEG, (e) GS-PEG-TTA1, (f) DNA/GS-PEG-TTA1, (g) GS-PEG-TTA1/TAT and (h) DNA/GS-PEG-TTA1/TAT.

\subsection{Cytotoxicity Assay in Vitro}

The viability of the U251 cells measured by MTT assay was shown in Fig.4. After incubating with nanoparticles for $24 \mathrm{~h}$, the cell viability is decreasing with the nanoparticles concentration increased. As 
shown in Fig.4, viability of cells pretreated with GS-PEG-TTA1 and GS-PEG-TTA1/TAT NPs, taking $100 \mu \mathrm{g} / \mathrm{ml}$ as an example, are $81.27 \%$ and $72.37 \%$, lower than the GS-PEG group $90.57 \%$ obviously. When the concentration increased to $500 \mu \mathrm{g} / \mathrm{ml}$, the viability of cells was all lower than other concentrations respectively.

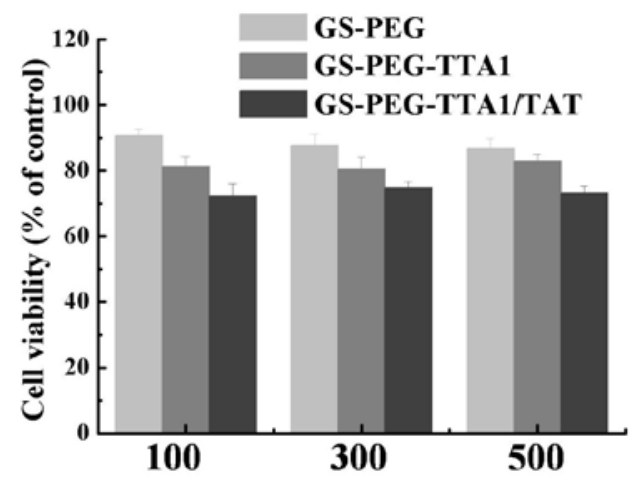

Figure 4. Normalized dose-response for cell viability of GS -PEG NPs, GS-PEG-TTA1 NPs, GS-PEG-TTA1/TAT NPs by MTT assay for 24h.Bars represent the corresponding standard deviations(n=6).

\subsection{In Vitro Cellular Uptake of Nanoparticles}

After incubated with the U251 cell, a part of the nanoparticles could penetrate cell membrane. Because the nanoparticles and plasmid were respectively labeled with FITC and PI, the fluorescence intensity in cell could represent the penetrating capacity of the nanoparticles. As shown in Fig.5, the colocalization of nanoparticles with plasmid produced a yellow fluorescence in merged images. GS-PEG-TTA1 group has a more intensity fluorescence than GS-PEG group, at the same time, GS-PEG-TTA1/TAT more easily crosses the cell membrane than others as TAT has the adventage of helping nanoparticles cross cell membrane. What is more, Fig.6 can indicate the tendency as described above more effectively with a quantitative determination method. Fig.6A and Fig.6B indicated that along with the co incubate time extension, more fluorescence can be detected as more nanoparticles uptook by U251 cells. Both shown in Fig.6A and Fig.6B, the mean fluorescence intensity displayed nanoparticles modified by TTA1 were easier taken up by U251 cells than those without modified. As compared in Fig.6C, after incubated with cell for $1 \mathrm{~h}$ and $2 \mathrm{~h}$, the proportions of penetrate cell membrane of nanoparticles modified by TTA1 are $(46.57 \pm 3.23) \%$ and $(71.47 \pm 2.54) \%$, having an apparent superiority relative to the other group without TTA1. What is more, with the assistance of TAT, FITC-GS-PEG-TTA1/TAT has a more efficient penetrate capability, $(79.16 \pm 3.75)$ and $(81.73 \pm 1.54) \%$. Those differences were all statistically significant $(P<0.05)$.

\subsection{In Vitro Transgene Expression}

As pitched on as reporter gene, luciferase plasmid pGL3 was carried out to test the expression efficiency after delivered by nanoparticles to U251 and Hela cells in vitro. Shown in Fig. 7, in both U251 and Hela cells, DNA/GS-PEG NPs and naked DNA were all transfected barely. However, after decorated with TTA1, the quantitative value of DNA/GS-PEG-TTA1 in U251 cells is $422 \mathrm{RLU} / \mathrm{mg}$, which is 1.55 -fold higher than transfected in Hela cells and 4.79-fold higher than DNA/GS-PEG in U251 cells. The nanoparticles comodified with TTA1 and TAT, both in U251 cells and Hela cells, have higher transfection efficiency (467 RLU/mg and $325 \mathrm{RLU} / \mathrm{mg}$ ) than any other nanoparticles. In addition, it can be found that the former, which is transfected in U251 cells is higher remarkable than in Hela cells. $(p<0.05)$. 


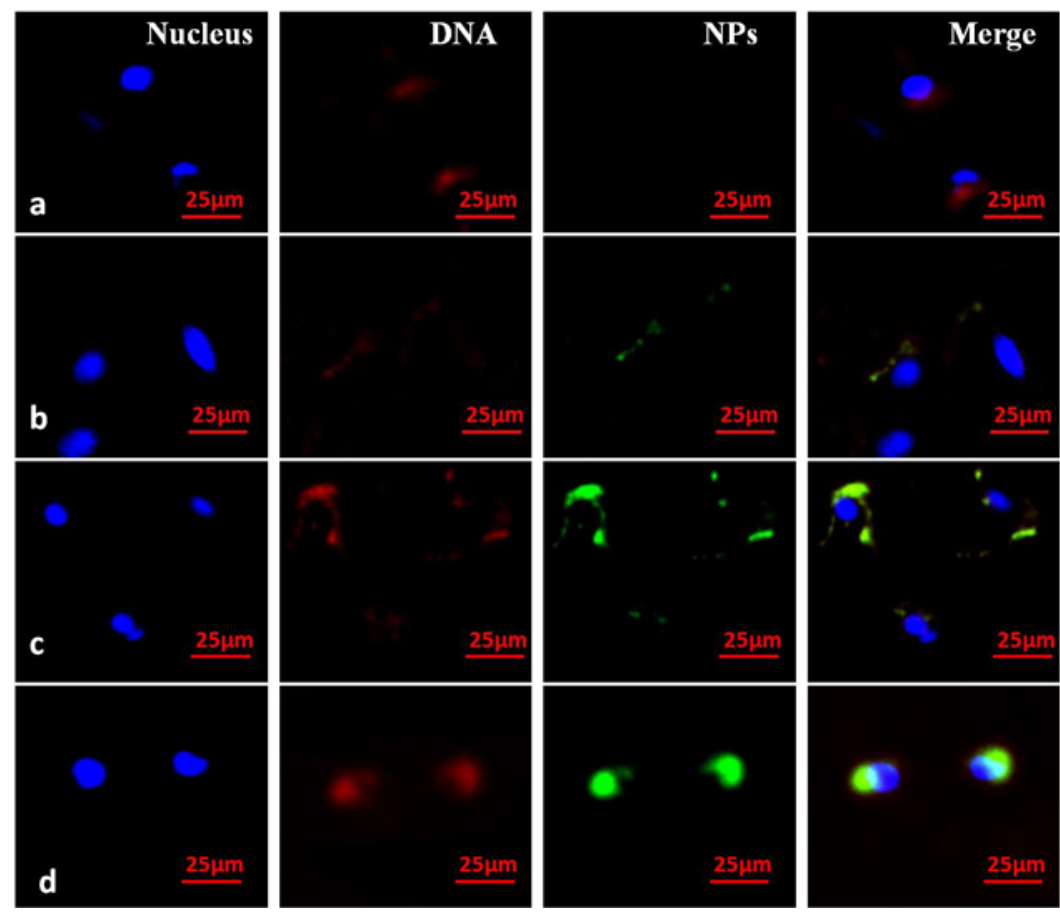

Figure 5. Fluorescence Microscope images of U251 cell incubated with (a) naked DNA, (b) DNA/GS-PEG, (c) DNA/GS-PEG-TTA1, (d) DNA/GS-PEG-TTA1/TAT for $4 \mathrm{~h}$.
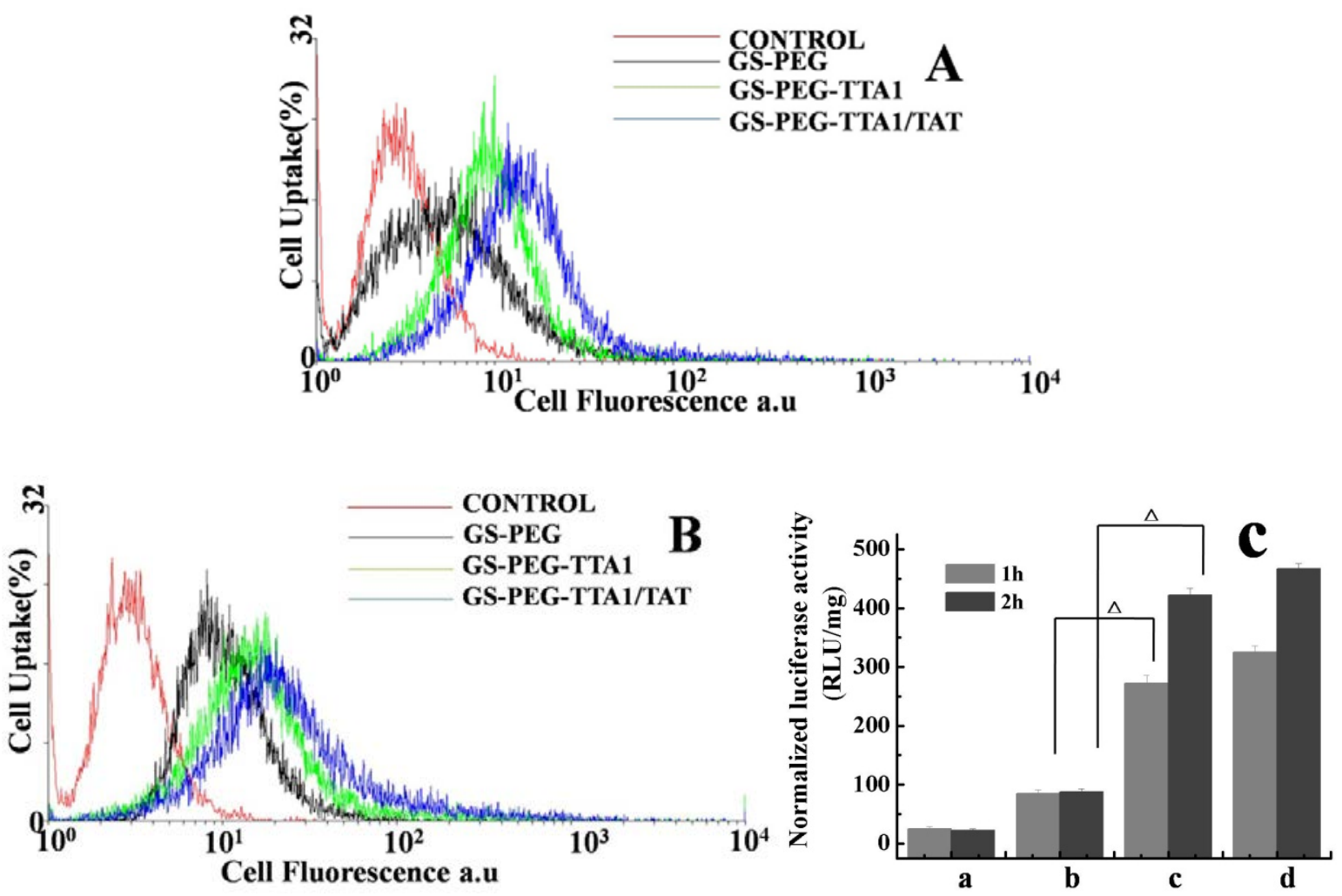

Figure 6. (A, B) Flow cytometery evaluate the cellular uptake efficiency after incubated with various GS NPs in $1 \mathrm{~h}$ and $2 \mathrm{~h}$. (C) Cellular internalization of being incubated with various GS NPs for $1 \mathrm{~h}$ and $2 \mathrm{~h}$. (a) Control, (b) FITC-GS-PEG, (c) FITC-GS-PEG-TTA1, (d) FITC-GS-PEG-TTA1/TAT

Notes: $\Delta P<0.05$ indicate a statistically significant difference; means \pm standard deviations are shown $(\mathrm{n}=5)$. 


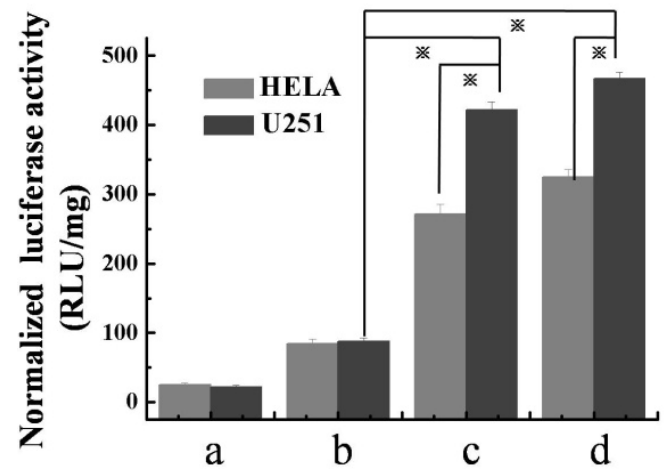

Figure 7. Nanoparticle mediated luciferase gene transfection in U251 cell and Hela cells. (a) Naked DNA, (b) DNA/GS-PEG, (c) DNA/GS-PEG-TTA1, (d) DNA/GS-PEG-TTA1/TAT

Notes: $* \quad P<0.05$ indicate a statistically significant difference; means \pm standard deviations are shown $(\mathrm{n}=5)$.

\section{Discussion}

As is known to all that Glioblastoma is one of the most malignant brain tumors, causing death within two years despite maximal tumor resection and concurrent radio-chemotherapy[18]. Therefore, there are uninterrupted endeavor of trying to find an effectively therapeutic method to inhibit the bloodcurdling tumor domestic and overseas $[19,20]$. There are increasing reports that gene therapy has distinct effect and bright future. However, all cancer gene therapy approaches rely on a sufficient level of delivery of the anticancer agent into target cells. Thus, enhancement of delivery to target cells, and reduction of delivery to non-target cells, in an approach called transductional targeting, is attractive [21]. As reported [22], TTA1, a kind of RNA aptamer, was previously isolated using systematic evolution of ligands by exponential enrichment (SELEX) against tumor cells and purified tenascin-C [23]. This aptamer has improved characteristics relative to an initial tenascin-C aptamer, discovered in a SELEX experiment targeting U251 glioblastoma cells [24]. TTA1 binds with a dissociation constant of $5 \mathrm{nmol} / \mathrm{L}$ to the fibrinogen-like domain of tenascin-C. Those all provided significantly experimental and theoretical foundation.

The gene vectors introduced in our report all have distinguishing features of average size, dispersive and spherical. As known to all, the surface charge and the particle size of gene-vectors are of very important factors. Those significant characters may affect the nanoparticles capacity of crossing the cell membrane noticeable. It had been reported that the density of TAT is to be an important factor in cellular delivery[25]. However, an excess of TAT would contribute to the cellular delivery hardly. The ratio of TAT/GS NPs in this experiment was $1.58 \mu \mathrm{mol} / \mathrm{g}$, as described by Yin Pei [17] in his article that the ratio among three ratios $(0.79 \mu \mathrm{mol} / \mathrm{g}, 1.58 \mu \mathrm{mol} / \mathrm{g}$ and $3.16 \mu \mathrm{mol} / \mathrm{g})$ does not significantly influence the diameter and surface charge of peptide-GS NPs. TTA1 is a kind of RNA aptamer, whose molecular weight is so small that cannot impact the particle size obviously, thus the ratio of TTA1/GS was $2 \mathrm{nmol} / \mathrm{mg}$ in this experiment. Fig.1 (a) and (b) prove that with the ratio of TAT/GS and TTA1/GS NPs as above, TAT and TTA1 both could be decorated to the GS NPs via a disulfide bond between the amidogen Sulfhydrylated via SPDP and free sulfhydryl on TAT or TTA1. As labeled with FITC differently, the difference between GS-PEG-TTA1 NPs and GS-PEG-TTA1/TAT NPs did not have any statistical significance. The sizes and surface charge were very important to the whole experiment. As shown in Fig.3, the size of basic nanoparticles is only $170.95 \mathrm{~nm}$, and those with modification of TAT and TTA1 are all bigger than that, but the biggest one is approaching $250 \mathrm{~nm}$, which is also as small as crossing the BBB[26]. The nanoparticles had positively charged surface with a measured zeta potential of $25.12 \mathrm{mV}$ for GS NPs and $3.12 \mathrm{mV}$ and $3.33 \mathrm{mV}$ for GS-PEG NPs and GS-PEG-TTA1 NPs, respectively. The positively charged decreased obviously after decorated with PEG and TTA1, as vast amidogen occupied by them. Nevertheless, peptides TAT was used to modify the GS NPs and assist it to have even more positively 
charged (Fig.3). As a kind of gene vector, the ability of carrying gene is having a great importance. In order to reach the condition of having less aggregated and desirable positive surface charge, we chose the ratio of NPs/DNA as 200/1 as the method described previously[17]. Though the particle size increased and the positively charged was decreased (Fig.3), the character of the nanoparticles was also available [26].

U251, a kind of spongioblastoma cell, in which tenascin-C can be over expressed was selected to detect the targeting ability of aptamer TTA1 in this experiment. Before that, in vitro cytotoxicity assay of the nanoparticles was employed at first. Formazan absorbance detected by microplate reader can represent the quantity of viable cell. As shown in Fig.4, the cell viability of all groups is above $72 \%$. The lowest viability group (in Fig.4) is GS-PEG-TTA1/TAT NPs of $500 \mu \mathrm{g} / \mathrm{ml}$, which also maintains the quantity of viable cell at above $70 \%$. Compared with the inorganic nanoparticles, such as Au NPs, CNTs and so on, GS NPs had higher median lethal dose [27], the modified GS NPs could be considered comparatively non-cytotoxic. It is also indicated in Fig.4 that the viability of cells pretreated with nanoparticles in the $100 \mu \mathrm{g} / \mathrm{ml}$ concentration was higher than other concentrations, thus this concentration was selected and used in cellular uptake experiments.

As introduced above, TTA1 had a magic ability of targeting combined with tenascin-C. In this article, as shown in Fig.5, after incubated with U251 cell for 4h, the FITC-labeled nanoparticles could pierce the cell membrane partially, and the PI- labeled DNA also had well internalization. After contrasted with each other, it could be found that naked DNA and DNA/GS-PEG had very weak ability of crossing the cell membrane as the red fluorescence in Fig.5a and b is so feeble that it hardly could be seen. However, those two groups decorated with TTA1 had evidently great internalization efficiency than others, just as the same result indicated in Flow cytometery detection. Fig.6A or B both demonstrated GS-PEG-TTA1 and GS-PEG-TTA1/TAT had stronger ability of internalizing accompanying with PI- labeled DNA than nanoparticle without TTA1. In Fig.6C, quantitative analysis of the internalization capacity of the nanoparticles showed that after cocultured with the cells for $2 \mathrm{~h}$, the cellular uptake index of GS-PEG-TTA1 was 1.06-fold higher than GS-PEG nanoparticles $(\mathrm{P}<0.05)$ and GS-PEG-TTA1/TAT was 1.36-fold higher than that $(\mathrm{P}<0.05)$. It was apparent that just the tremendous contribution of TTA1 of targeting ability impel the nanoparticles to access the tumor cells more efficiently. It also could demonstrate in Fig.5 and Fig.6 that GS-PEG-TTA1/TAT had higher ability of internalization than GS-PEG-TTA1 just because of the contribution of cationic peptide TAT as described in the literature [28-29].

The main mission of this experiment is to test whether the GS NPs would be a kind of duteous gene vector. Thus after cell uptaked by U251 cell, the gene delivered by nanoparticles were designed to test whether it can express in the tumor cells. Fig.7 indicated that with the help of TTA1, DNA/GS-PEG-TTA1 and DNA/GS-PEG-TTA1/TAT both had higher expression efficiency than DNA/GS-PEG and naked DNA in the two kinds of tumor cells. What is more, DNA/GS-PEG-TTA1 can express higher in U251 than in Hela $(\mathrm{p}<0.05)$. It can be explained as the tenascin-C cannot express in the normal cells, but it plays a role in tumor progression, especially in the glioblastoma [15]. In other words, just as tenascin-C had a high-efficiency expression in U251 cells that DNA delivered by GS-PEG-TTA1 express obviously more in U251 than in Hela as shown in Fig.7 ( $<<0.05)$.

Besides the helping of the specially targeting capability of TTA1, the internalization process would be affected obviously by the electrostatic interactions between cell membrane and cationic nanoparticles, as the surface of the cells is an anionic membrane[30]. In order to facilitate the uptake of the nanoparticles, the CPPs were great choice. Peptide TAT (YGRKKRRQRRR) is the protein transduction domain from the transcriptional activator TAT protein of the human immunodeficiency virus type-1 (HIV-1)[31-32]. Though the specific mechanism of how the cationic peptides crossing the cell membrane is not very clear, it can help the vectors internalize seriously as reported[33-34]. As shown in Fig.3, the surface charge of GS-PEG-TTA1/TAT leaped after modified with TAT, but the particle size increased feebly, relatively. Just because of the positive charge around the nanoparticles, it could cross the cell membrane more effectively. Large amounts of kelly fluorescent orbiting the blue center which are the special effect of merging the green fluorescent and red fluorescent (Fig.5). By calculation to compare the distinction between different nanoparticles, Fig.6 showed that GS-PEG-TTA1/TAT had the incontrovertible dominant position in helping uptake of nanoparticles by cells. No matter whether it is incubated with cells for $1 \mathrm{~h}$ or $2 \mathrm{~h}$, there is clear evidence that GS-PEG-TTA1/TAT can enter the cells more availably than GS-PEG-TTA1, not to speak the GS-PEG. On account of the above results, the results in Fig.7, DNA/GS-PEG-TTA1/TAT has the absolute advantage of delivering more DNA/GS composite into cells 
and expressing in the cells under the same condition with the other groups, which seems to be a matter of course.

\section{Conclusion}

The fundamental purpose of this article is to structure a novel gene vector which has the characteristics of being small enough, hypotoxicity, and efficacious delivering DNA into targeting tumor cells and expressing in the cells. It was demonstrated in this article that gelatin-siloxane nanoparticles (GS NPs) have the significant characteristic of being innocuous, small, covered with positive charge and even have been modified with PEG, TTA1, and TAT. The modified nanoparticles, GS-PEG-TTA1/TAT, not only have the ability of crossing the cell membrane with DNA accompanied effectively, but also could target the U251 cells and impel the DNA express right in the cells with the help of aptamer TTA1. Therefore, the formulations of compounding the novel gene vector DNA/GS NPs could be a promising solution for in vivo experiments of delivering gene to penetrate the BBB for gene therapy the Glioblastoma.

Acknowledgment. This work was financially supported by the National Natural Science Foundation of China (81172394), and the National Natural Science Foundation of China (30970733).

\section{Reference}

1. Yi D, Hua TX, Lin HY, et al. Antitumor treatment efficacy by targeting epidermal growth factor receptor and vascular endothelial growth factor receptor-2 in an orthotopic human glioblastoma model. J Neurooncol. 2011; 104(1):93-101.

2. Gwak HS, Youn SM, Kwon AH, et al. ACNU-cisplatin continuous infusion chemotherapy assalvage therapy for recurrent glioblastomas: phase II study. J Neurooncol. 2005;75(2):173-180.

3. Liu L, Guo K, Lu J, et al. Biologically active core/shell nanoparticles self-assembled from cholesterol-terminated PEG-TAT for drug delivery across the blood-brain barrier. Biomaterials. 2008;29(10):1509-17.

4. Chen YZ, Yao XL, Tabata Y, Nakagawa S, Gao JQ. Gene carriers and transfection systems used in the recombination of dendritic cells for effective cancer immunotherapy. Clin Dev Immunol. 2010;2010:565643.

5. Vetrini F, Ng P.Gene therapy with helper-dependent adenoviral vectors: current advances and future perspectives. Viruses. 2010;2(9):1886-917.

6. Fulci G, Chiocca EA.The status of gene therapy for brain tumors. Expert Opin Biol Ther. 2007;7(2):197-208.

7. Tros de Ilarduya C, SunY, Düzgüneş N. Gene delivery by lipoplexes and polyplexes. Eur J Pharm Sci. 2010;40(3):159-70.

8. Tian XH, Lin XN, Wei F, et al. Enhanced brain targeting of temozolomide in polysorbate- 80 coated polybutylcyanoacrylate nanoparticles. Int J Nanomedicine. 2011;6:445-452.

9. Kreuter J, Ulbrich K, Hekmatara T, Herbert E. Transferrin-and transferrin-receptor-antibody-modified nanoparticles enable drug delivery across the blood-brain barrier (BBB). Eur J Pharm Biopharm. $2009 ; 71(2): 251-256$

10. R. Fischer, M. Fotin-Mleczek, H. Hufnagel, R. Brock, Break on through to the other side-biophysics and cell biology shed light on cell-penetrating peptides, Chembiochem.2005;6(12):2126-2142.

11. Ko HY, Choi KJ, Lee CH, Kim S.A multimodal nanoparticle-based cancer imaging probe simultaneously targeting nucleolin, integrin $\alpha \mathrm{v} \beta 3$ and tenascin-C proteins. Biomaterials. 2011;32(4):1130-8

12. Wurster SE, Bida JP, Her YF, Maher 3rd LJ. Characterization of anti-NF-kappaB RNA aptamer-binding specificity in vitro and in the yeast three-hybrid system. Nucleic Acids Res. 2009;37(18):6214-6224.

13. Li Y, Lee HJ, Corn RM. Fabrication and characterization of RNA aptamer microarrays for the study of protein-aptamer interactions with SPR imaging. Nucleic Acids Res. 2006;34(22):6416-6424.

14. Midwood KS, Hussenet T, Langlois B, Orend G.Advances in tenascin-C biology. Cell Mol Life Sci. 2011;68(19):3175-99

15. Kim MY, Kim OR, Choi YS, Lee H, Park K, Lee CT, Kang KW, Jeong S. Selection and characterization of tenascin C targeting peptide. Mol Cells. 2012;33(1):71-77 
16. Wang ZY., Zhao Y, Ren L, et al. Novel gelatin-siloxane nanoparticles decorated by Tat peptide as vectors for gene therapy[J]. Nanotechnology. 2008, 19(44):445103.

17. Yin P, Wang J, Ren L, et al. Conjugation of membrane-destabilizing peptide onto gelatin-siloxane nanoparticles for efficient gene expression, Materials Science \& Engineering C-Materials for Biological Applications.2010;30(8):1260-1265.

18. Motegi H, Kamoshima Y, Terasaka S, et al. A novel adherent culture method of glioblastoma cells expressing CD133 using collagen-1-coated plates. Hokkaido Igaku Zasshi. 2012;87(4-5):147-151.

19. Kurozumi K, Ichikawa T, Onishi M, et al. Cilengitide treatment for malignant glioma: current status and future direction. Neurol Med Chir (Tokyo). 2012;52(8):539-547.

20. Wang L, Zhao H, Cui K, et al. Identification of novel small-molecule inhibitors of glioblastoma cell growth and invasion by high-throughput screening. Biosci Trends. 2012; 6(4):192-200.

21. Ranki T, Hemminki A. Serotype chimeric human adenoviruses for cancer gene therapy. Viruses. 2010;2(10):2196-212.

22. Hicke BJ , Stephens AW, Gould T, et al. Tumor targeting by an aptamer. The Journal Of Nuclear Medicine.2006,47(4):668-768.

23. Hicke BJ, M arion C, Chang YF, et al. Tenascin-c aptamers are generated using tumor cells and purified protein. J Biol Chem. 2001;276:48644-48654.

24. Daniels DA, C hen H, Hicke BJ, Swiderek KM, Gold L. A tenascin-c aptamer identified by tumor cell SELEX: systemat ic evolution of ligands by exponential enrichment. Proc Natl Acad Sci USA.2003;100(26):15416-15421.

25. Eguchi, A., Akuta, T., Okuyama, H., et al. Protein transduction domain of HIV-1 TAT protein promotes efficient delivery of DNA into mammalian cells. J. Biol. Chem. 2001;276(28), 26204-26210.

26. Agarwal A, Lariya N, Saraogi G, et al. Nanoparticles as novel carrier forbrain delivery: a review. Curr Pharm Des, 2009,15(8): 917-925.

27. Zhi Ping Xu, Qing Hua Zeng, Gao Qing Lu, Ai Bing Yu. Inorganic nanoparticles as carriers for efficient cellular delivery. Chemical Engineering Science. 2006, 61,(3):1027-1040

28. Paul A, Binsalamah ZM, Khan AA, et al. A nanobiohybrid complex of recombinant baculovirus and Tat/DNA nanoparticles for delivery of Ang-1 transgene in myocardial infarction therapy. Biomaterials. 2011;32(32):8304-8318.

29. Lee SJ, Yoon SH, Doh KO. Enhancement of gene delivery using novel homodimeric tat peptide formed by disulfide bond. J Microbiol Biotechnol. 2011;21(8):802-807.

30. Chung TH, Wu SH, Yao M, et al. The effect of surface charge on the uptake and biological function of mesoporous silica nanoparticles in 3T3-L1 cells and human mesenchymal stem cells. Biomaterials. 2007;28(19):2959-66.

31. Liu L, Guo K, Lu J, et al. Biologically active core/shell nanoparticles self-assembled from cholesterol-terminated PEG-TAT for drug delivery across the blood-brain barrier. Biomaterials. 2008;29(10):1509-17.

32. Schwarze SR, Ho A, Vocero-Akbani A, Dowdy SF. In vivo protein trans-duction: delivery of a biologically active protein into the mouse. Science. 1999;285(5433):1569-1572.

33. Arjen van den Berg and Steven F Dowdy. Protein transduction domain delivery of therapeutic macromolecules. Current Opinion in Biotechnology. 2011, 22(6):888-893.

34. Qin Y, Chen H, Yuan W, et al. Liposome formulated with TAT-modified cholesterol for enhancing the brain delivery. Int J Pharm. 2011;419(1-2):85-95. 